\title{
Image analysis on the flexural behaviors of RC beams with artificial damage and the effects of repair
}

\author{
Kota Matsue ${ }^{1, *}$, Takashi Matsumoto ${ }^{2}$, Ko Kakuma ${ }^{3}$, and Hiroaki Nishi $^{3}$ \\ ${ }^{1}$ Hokkaido University, Graduate School of Engineering, Kita 13 Nishi 8, Kita-ku, Sapporo, Japan \\ ${ }^{2}$ Hokkaido University, Faculty of Engineering, Kita 13 Nishi 8, Kita-ku, Sapporo, Japan \\ ${ }^{3}$ Civil Engineering Research Institute for Cold Region, 1-3 Hiragishi, Toyohira-ku, Sapporo, Japan
}

\begin{abstract}
In cold and snowy regions, frost damage happened in an RC bridge deck slab leads to horizontally layered cracks, which results in the disintegration of cover concrete. Since this deterioration mode occurs inside an RC slab, it is usually difficult to detect them. It is possible that the slab performances have already decreased when the deteriorations appear on the exterior surface. As a preliminary step towards the establishment of a method for evaluating the structural performance of such a damaged RC slab, the purpose of this paper is to investigate the relationship between deteriorations and structural behaviors and to study the effect of an epoxy injection repair. For simplification, RC beams are prepared with artificial cracks that simulate deteriorations and these were made by the use of expansive agent. These beams are tested in flexure. Since the damage is volumetric, image analysis is useful to capture the two-dimensional deformations. It is shown that with different deterioration depths that is a part of the compression side of the beam or full of the height, there are differences about the deformation behaviors, the repair effects and the distributions of maximum and minimum principal strain.
\end{abstract}

\section{Introduction}

$\mathrm{RC}$ bridge deck slabs deteriorate due to several causes. When fatigue causes deteriorations, cracks are formed first in the transverse direction and later also in the longitudinal direction on the bottom surface of RC slabs. In the final moment, punching shear fatigue failure is observed [1-2]. In cold and snowy regions, frost damage leads to horizontally layered cracks, which results in the disintegration of cover concrete. With frost damage, fatigue load capacity decreases due to the effective slab thickness decrease. Since these deterioration modes occur inside RC slabs, it is usually difficult to detect them. It is possible that the slab performances have already decreased when the deteriorations appear on the exterior surface. As a preliminary step towards the establishment of a method for evaluating the structural performance of such damaged RC slabs, the purpose of this paper is to investigate the relationship between deteriorations and structural behaviors and to study the effect of an epoxy injection repair. For simplification, RC beams are prepared with artificial cracks that simulate deteriorations, and these beams are tested in flexure. Since the damage is volumetric, image analysis is useful to capture the two-dimensional deformations [3]. From a surface displacement field, maximum and minimum principal strain fields are calculated. Based on the strain fields, the deformation behaviors of RC beams with artificial cracks will be evaluated for non-repair and repair cases.

\section{Bending test of RC beams}

\subsection{Specimens}

Fig. 1 shows specimen dimensions, artificially cracked regions, and epoxy injection points. The length, width, and height of specimens are $1700 \mathrm{~mm}, 100 \mathrm{~mm}$, $200 \mathrm{~mm}$, respectively. The span length between supports is $1400 \mathrm{~mm}$, while the shear and flexural span of specimens are $500 \mathrm{~mm}$ and $400 \mathrm{~mm}$, respectively. Shear span ratio is 2.5. Table 1 shows the names of six specimens. Three specimens of D-series have artificial cracks, while three specimens of RD-series have artificial cracks in the same way as those of D-series and they were repaired with epoxy injection. Artificial cracks were made by the use of expansive agent which was mixed in fresh concrete of the regions. Before tests, local distributed cracks and pop-outs of concrete were observed in the regions. Epoxy injections were conducted at four points which were located $\pm 100 \mathrm{~mm}$ and $\pm 300 \mathrm{~mm}$ from the center of each specimen. After drilling $170 \mathrm{~mm}$ deep from the top surface, epoxy was injected into the specimens to fill the internal cracks artificially made.

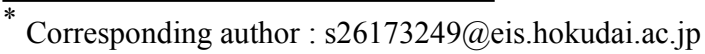




\subsection{Loading conditions}

Fig. 1 shows bending test setups. The specimens were tested under four-point flexure under load control before

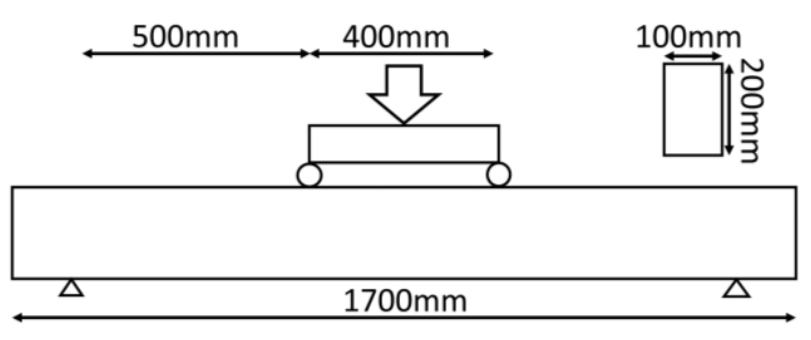

The points of epoxy injection (200mm interval)

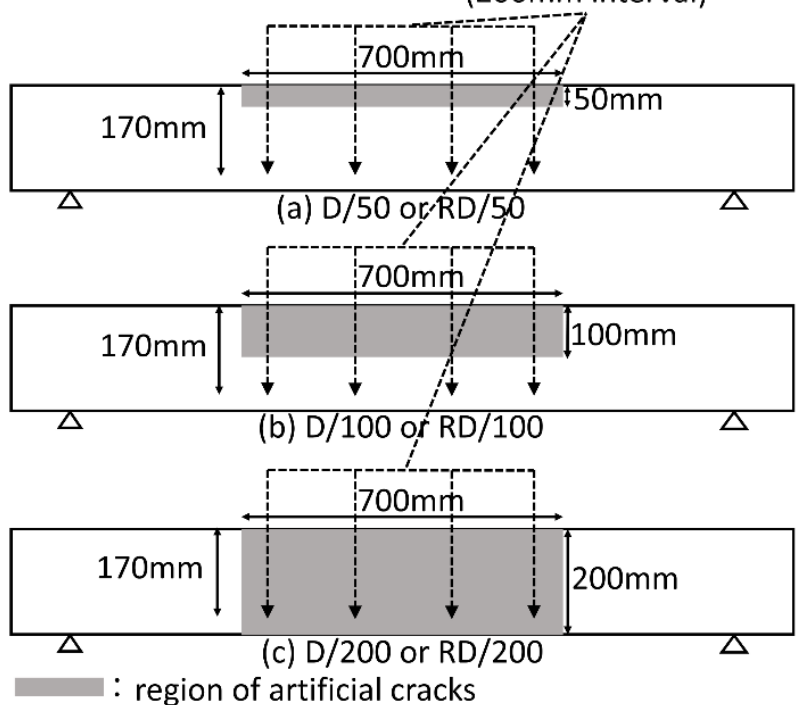

Fig. 1. Test setup: specimen dimensions, artificial damage and repair.

Table 1. Specimens names.

\begin{tabular}{|c|c|c|}
\hline $\begin{array}{c}\text { Damage } \\
\text { depth }(\mathrm{mm})\end{array}$ & Damage only & $\begin{array}{c}\text { Damage } \\
\text { and repair }\end{array}$ \\
\hline $50 \mathrm{~mm}$ & $\mathrm{D} / 50$ & $\mathrm{RD} / 50$ \\
\hline $100 \mathrm{~mm}$ & $\mathrm{D} / 100$ & $\mathrm{RD} / 100$ \\
\hline $200 \mathrm{~mm}$ & $\mathrm{D} / 200$ & $\mathrm{RD} / 200$ \\
\hline
\end{tabular}

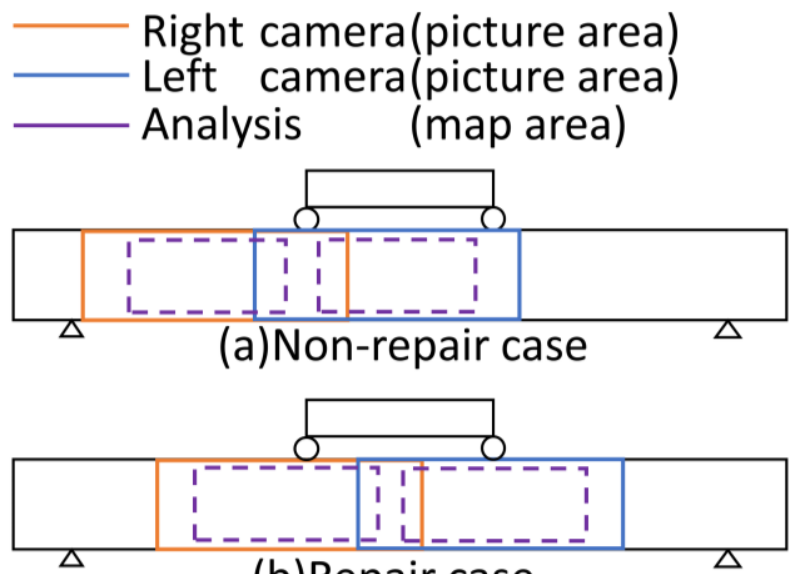

(b) Repair case

Fig. 2. The target area of camera and analysis.

the occurrences of flexural cracks and under displacement control after the occurrences. Steel plates were placed at the loading and support points. Between the specimen and the steel plate, fresh plaster was placed and hardened to fill the gap. In addition to image analysis, load, displacement, and surface strain (contact gauge) were measured.

\section{Method of image analysis}

\subsection{Image capture}

Image capture was conducted with a digital camera, Nikon D3100, which can capture image of $4608 \times 3072$ pixels. Fig. 2 shows the target of image analysis. Random pattern was made by using a spray so that digital image correlation method could be used for concrete surface. At first, image capture was conducted before loading, and later at every $10 \mathrm{kN}$ until the specimen shows yielding. After yielding, image capture was conducted at $1.5 \delta_{\mathrm{y}}$, $2.0 \delta_{\mathrm{y}}$, and $3.0 \delta_{\mathrm{y}}$, where $\delta_{\mathrm{y}}$ is the displacement at yielding.

\subsection{Image correlation}

An RGB color image is converted to a grayscale image, and furthermore to a binary image. Measurement points for image correlation are set as follows. Fig. 2 shows the area of image analysis where measurement points are allocated. For D-series, measurement points of 24 points in the horizontal direction and 12 points in the vertical direction are placed at the interval of 200 pixels. On RD-series, measurement points of 23 points in the horizontal direction and 10 points in the vertical direction are placed at the interval of 200 pixels. Mask image $(128 \times 128$ pixels $)$ is clipped out around a measurement point of the image which is captured before loading, and region image $(256 \times 650$ pixels $)$ is similarly clipped out from the image which is captured during loading. The rectangular region image takes a long side in the vertical displacement direction so as to include the displaced measurement point. A value outside the clipped region was set to 0 (black), and an image $(700 \times 700$ pixels $)$ including clipped image was created. The cross correlation is calculated for the two images of the 
measurement point before and after deformation, and the position with the maximum cross correlation value is regarded as the measurement point after deformation. The displacement vector can be obtained by the positions of the measurement point before and after deformation.
The maximum principal strain and the minimum principal strain are calucuralted via the horizontal strain and the vertical strain from the horizontal and vertical displacement of four measurement points, where the four measurement points form a square element.

\subsection{Calculation of strain}

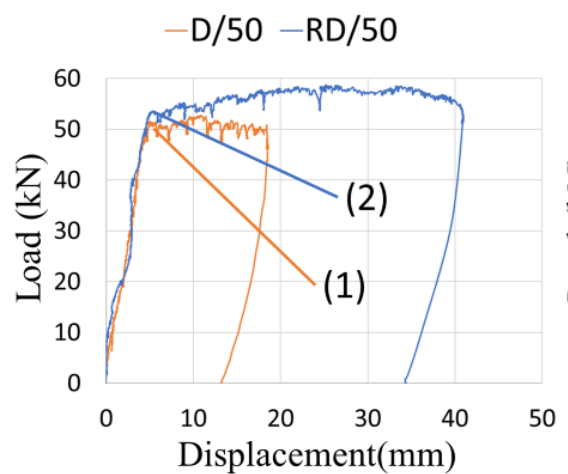

(a) $\mathrm{D} / 50, \mathrm{RD} / 50$

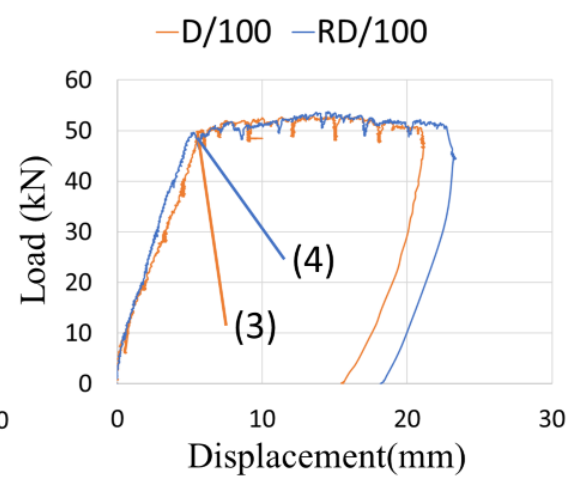

(b) $\mathrm{D} / 100, \mathrm{RD} / 100$

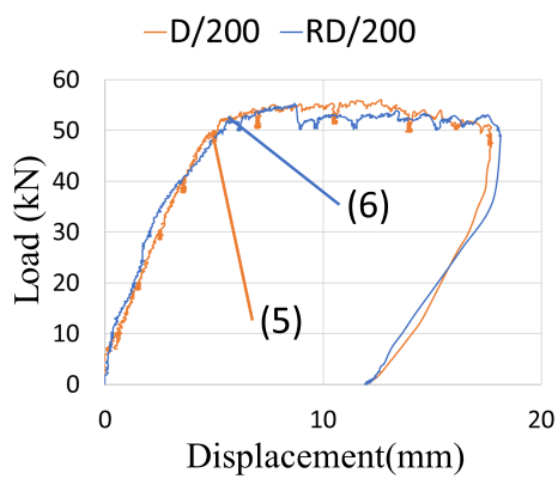

(c) $\mathrm{D} / 200, \mathrm{RD} / 200$

Fig. 3. Load-displacement relations.

Table 2. Load and displacement at yielding.

\begin{tabular}{|c|c|c|c|}
\hline Specimen & $\begin{array}{c}\text { Load at yielding } \\
(\mathrm{kN})\end{array}$ & $\begin{array}{c}\text { Displacement at yielding } \\
(\mathrm{mm})\end{array}$ & $\begin{array}{c}\text { Corresponding } \\
\text { point in Fig. 3 }\end{array}$ \\
\hline $\mathrm{D} / 50$ & 51.3 & 4.83 & $(1)$ \\
$\mathrm{RD} / 50$ & 52.9 & 5.78 & $(2)$ \\
\hline $\mathrm{D} / 100$ & 50.2 & 5.81 & $(3)$ \\
$\mathrm{RD} / 100$ & 49.1 & 5.78 & $(4)$ \\
\hline $\mathrm{D} / 200$ & 52.2 & 5.32 & $(5)$ \\
$\mathrm{RD} / 200$ & 51.6 & 5.63 & $(6)$ \\
\hline
\end{tabular}




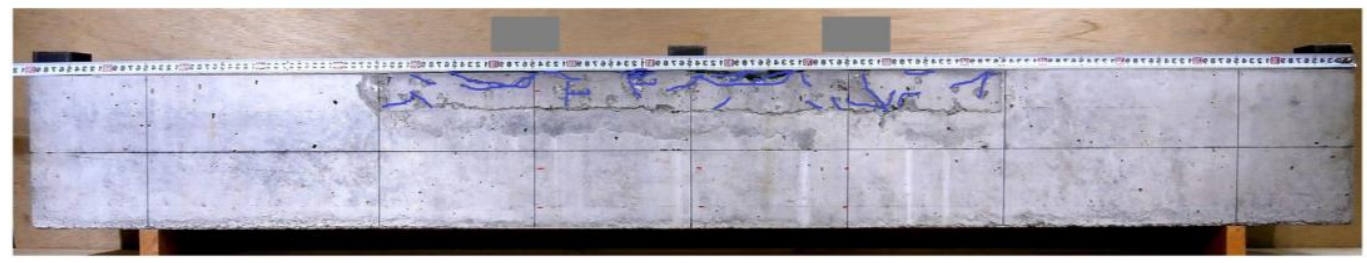

(a) Specimen before loading
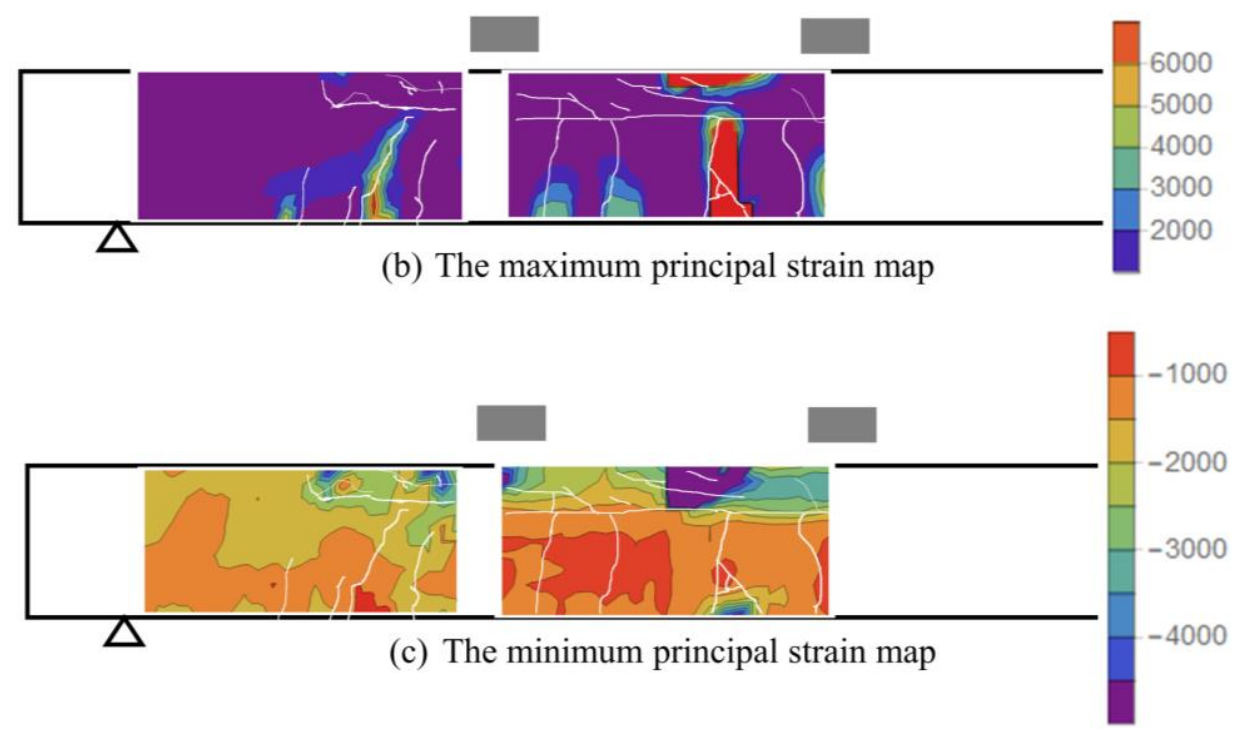

Fig. 4. D/50 specimen and strain maps $(51.3 \mathrm{kN}, 4.83 \mathrm{~mm})$.

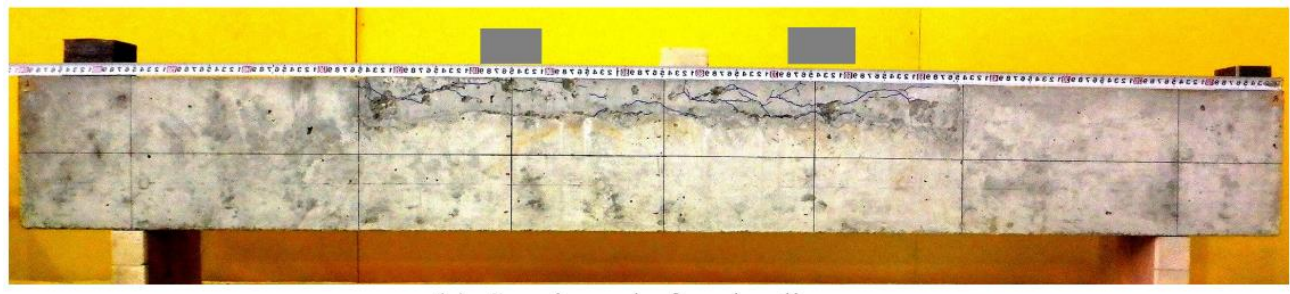

(a) Specimen before loading
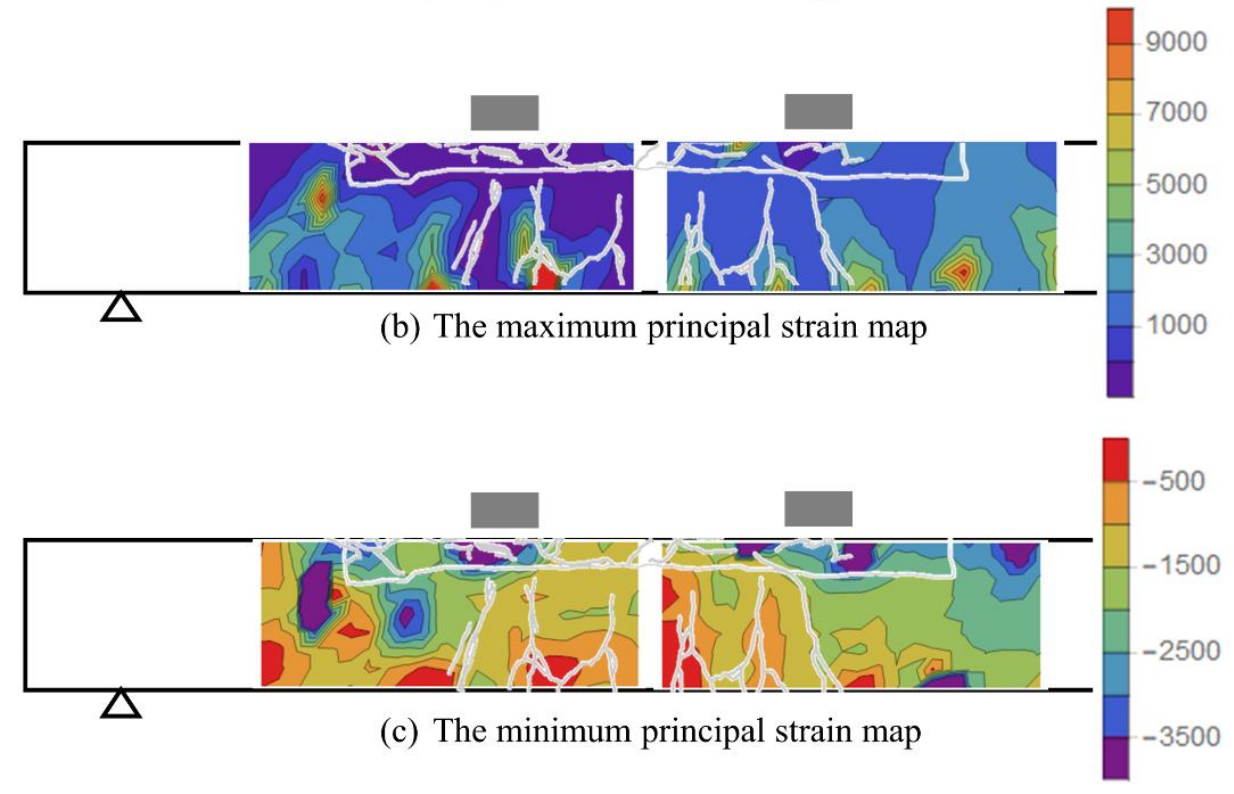

Fig. 5. RD/50 specimen and strain maps (52.9kN, 5.78mm). 


\section{Results of image analysis}

In this paper, the results of image analysis are shown for the moment when the specimens reached yielding. Fig. 3 shows load-displacement relations and Table 2 shows load and displacement at yielding.

There is no difference among the load-displacement curves associated with different damage depths. After the repair, the improvement of load capacity is observed for $\mathrm{RD} / 50$, but it is not observed for $\mathrm{RD} / 100$ and $\mathrm{RD} / 200$.

In the following figures of strain maps from Fig. 4 to Fig. 9, the sketches of all the cracks including the artificial cracks of the opposite side of each specimen after the loading are superimposed as a perspective view and are indicated by white lines. Similarly, in the picture of specimen before loading, the sketches of artificial cracks are shown by blue lines.

\subsection{D/50 and RD/50}

Regarding D/50, Fig. 4 shows the maximum principal strain map and the minimum principal strain map. In the maximum principal strain, a distribution corresponding to bending cracks occurring in the shear span and the flexural span is observed, and a distribution corresponding to a large open crack can be confirmed at the center of the flexural span. These are roughly corresponding to the crack sketches on the opposite side. On the compressive face in the flexural span, a strong horizontal distribution of the tensile opening can be seen. On the other hand, in the minimum principal strain, a distribution in the compressive direction can be seen in the upper part of the specimen matching the artificially damaged region (Fig. 1). Moreover, on the compressive face in the flexural span, a distribution of the compressive collapse at the same position as the maximum principal strain was observed.

Regarding RD/50, Fig. 5 shows the maximum principal strain map and the minimum principal strain map. In the maximum principal strain, a distribution due to bending cracks similar to $\mathrm{D} / 50$ is seen, and it seems that the distribution spreads over the shear span side. In the minimum principal strain, the distribution of compressive strain spreads in the direction from the loading point to the support. A distribution of the minimum principal strain matching the artificially damaged region observed in $\mathrm{D} / 50$ is not observed in $\mathrm{RD} / 50$. Some reduction of the minimum principal strain on the compressin face of the flexural span, which is arificially damaged region, is observed. In the shear span, the minimum principal strain spreads diagonally from the loading point towards support.

Based on above, the effects of the repair is partly recognized about the minimum principal strain distribution in the region of artificial cracks.

\subsection{D/100 and RD/100}

Regarding D/100, Fig. 6 shows the maximum principal strain map and the minimum principal strain map. In the maximum principal strain, a distribution due to opening cracks on the tension face is observed, and a strong distribution considered to be due to compression can be confirmed at the compression face of the flexural span. In the minimum principal strain, an overall distribution including a local and strong vertical distribution approximately corresponds to the artificially damaged region is seen.

Regarding RD/100, Fig. 7 shows the maximum principal strain map and the minimum principal strain map. In the maximum principal strain, similar to $\mathrm{D} / 100$, a distribution due to several opening cracks on the tension face can be confirmed, but a strong distribution on the compression face of the flexural span is not observed. In the minimum principal strain, a distribution spreading in the horizontal direction is observed at the compression face within the flexural span. However, this distribution does not coincide with the artificially damaged region, and no clear change is observed across the boundary of the artificially damaged region.

Based on the above, it is summarized that the local distribution of the minimum principal strain changed from vertical to horizontal spreading, and that the effects of the repair are partly recognized about the minimum principal strain distribution in the artificially damaged region.

\subsection{D/200 and RD/200}

Regarding D/200, Fig. 8 shows the maximum principal strain map and the minimum principal strain map. In the maximum principal strain, there is a strong and wide distribution: one in the shear span and two in the flexural spans. This distribution is unlike those of $\mathrm{D} / 50$ and $\mathrm{D} / 100$. The distribution progresses diagonally at shallow angles, not progressing vertically. Some parts are consistent with crack sketches. In the minimum principal strain, it is confirmed that a strong distribution around the loading plate takes place on the compression face of the flexural span.

Regarding RD/200, Fig. 9 shows the maximum principal strain map and the minimum principal strain map. A strong distribution of the maximum principal strain is seen in the shear span and the flexural span, which is significantly different from those of $\mathrm{RD} / 50$ and $\mathrm{RD} / 100$ and is similar to $\mathrm{D} / 200$. The crack sketch shows the occurrence of irregular cracks similarly to $D / 200$ before repair, but it does not coincide with the distribution of the maximum principal strain. In th e minimum principal strain, there is no distribution related to the artificially damaged region or cracks, and a strong distribution is observed on the compression face.

As a result of the artificially damaged region of the full depth, the maximum and minimum strain distribution are significantly different from those of normal RC beams. The effects of the repair are not observed in the maximum and minimum strain distribution, and no clear change is observed across the boundary of the artificially damaged region. 


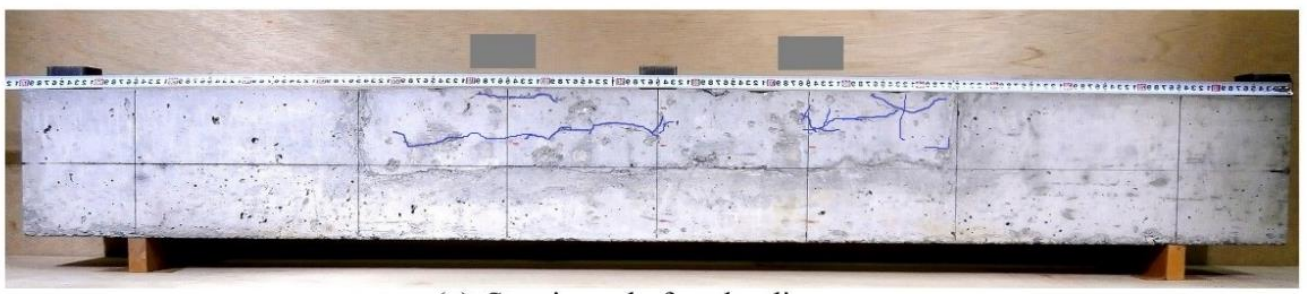

(a) Specimen before loading
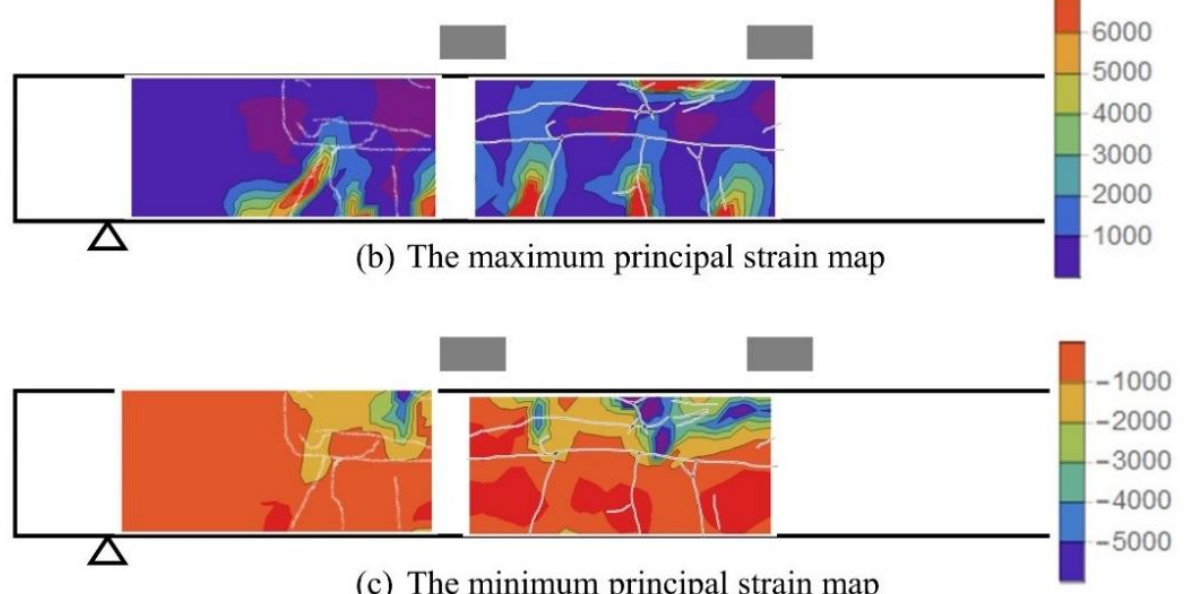

(c) The minimum principal strain map

Fig. 6. D/100 specimen and strain maps $(50.2 \mathrm{kN}, 5.81 \mathrm{~mm})$.

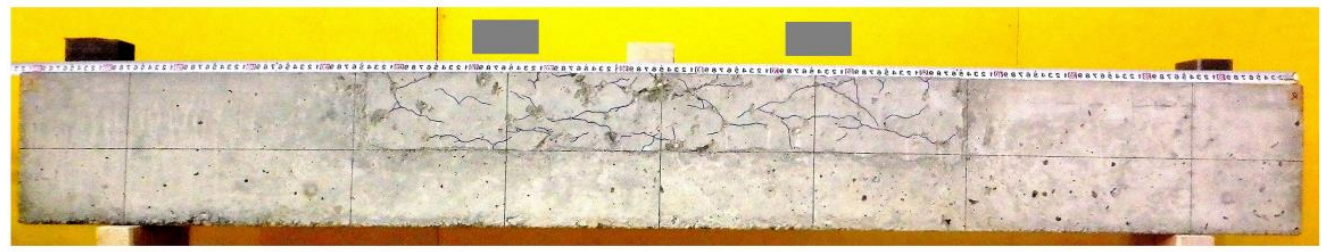

(a) Specimen before loading
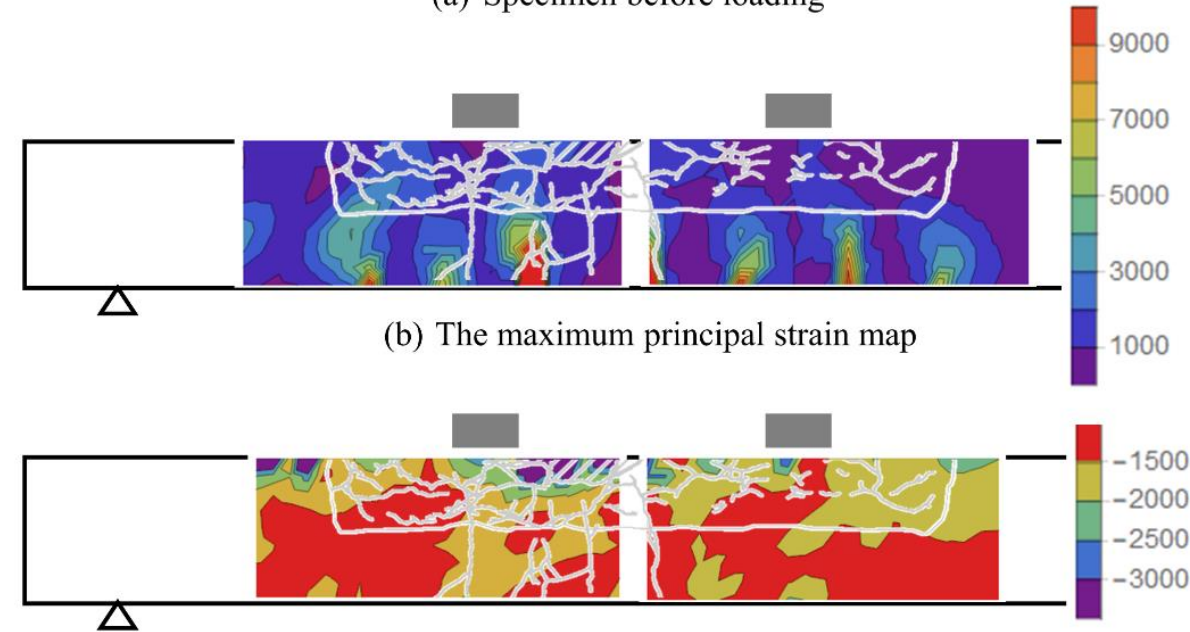

(c) The minimum principal strain map

Fig. 7. $\mathrm{RD} / 100$ specimen and strain maps $(49.1 \mathrm{kN}, 5.78 \mathrm{~mm})$. 


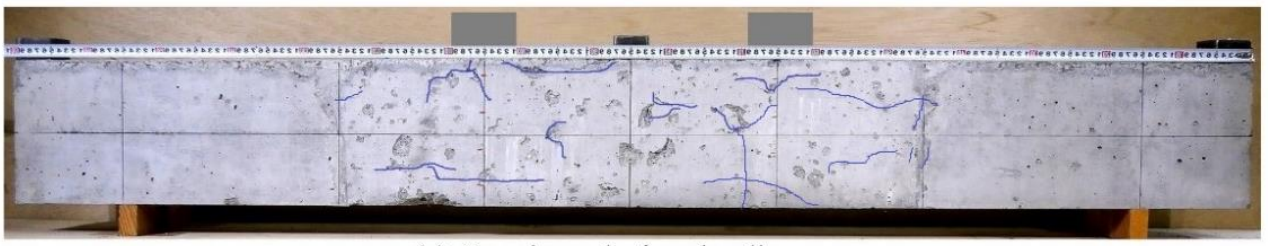

(a) Specimen before loading

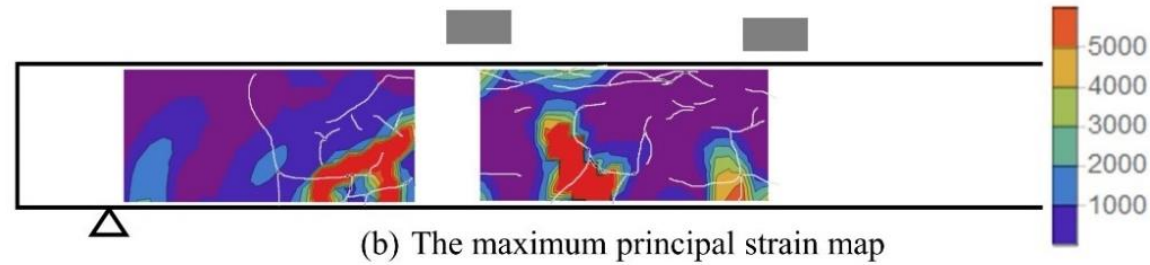

(b) The maximum principal strain map

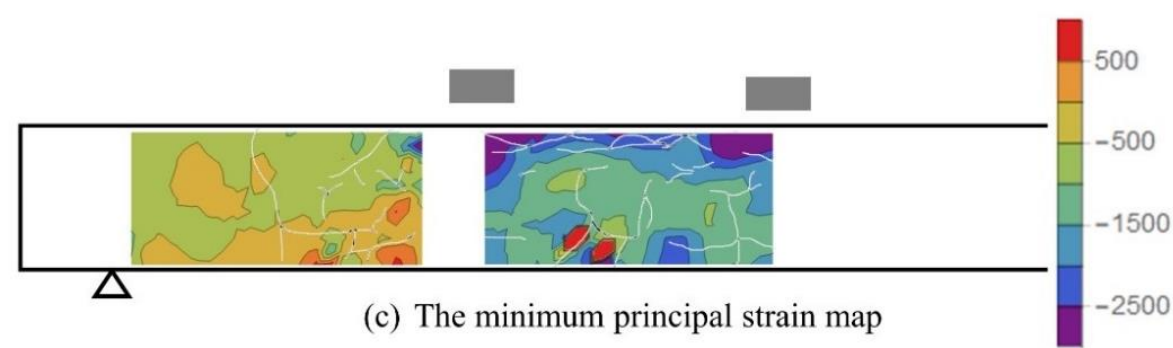

Fig. 8. D/200 specimen and strain maps $(52.2 \mathrm{kN}, 5.32 \mathrm{~mm})$.

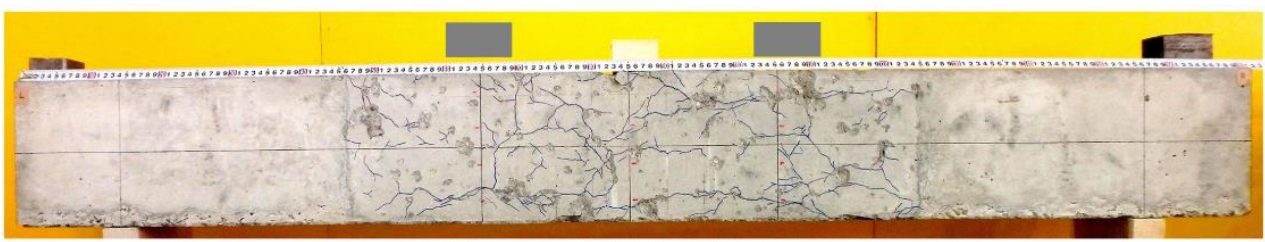

(a) Specimen before loading

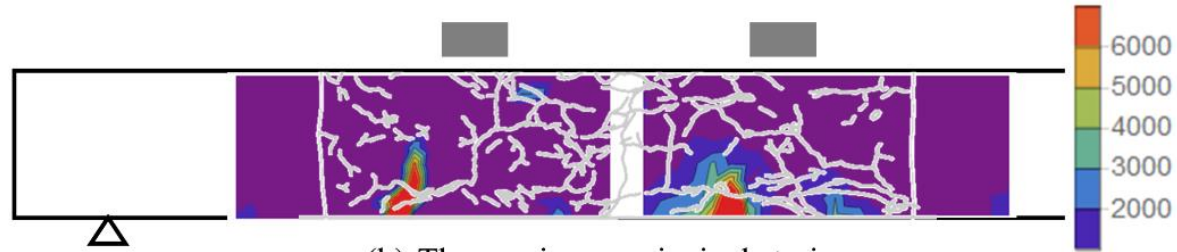

(b) The maximum principal strain map

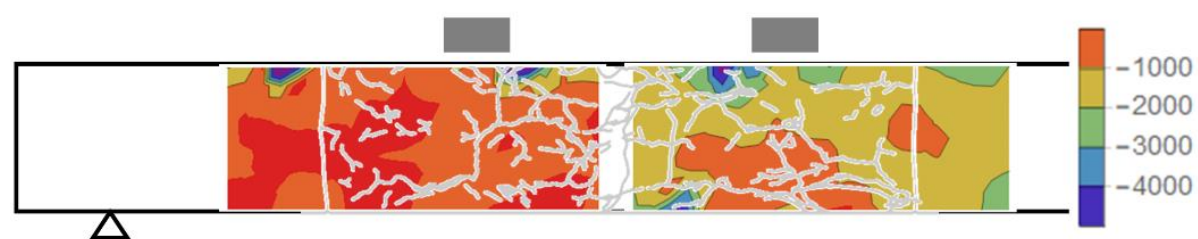

(c) The minimum principal strain map

Fig. 9. RD/200 specimen and strain maps (51.6kN, 5.63mm).

\section{Conclusions}

In this study, three RC beams are prepared with artificial cracks that simulate deteriorations, and additional three $\mathrm{RC}$ beams are prepared with artificial cracks and repaired with an epoxy injection method. These total six beams are tested in flexure and compared about the deformation behaviors and the repair effects by the use of image analysis. The results about the influence of artificial cracks and the effects of the repair are as follows:

When the depth of artificially damaged region is a part of the compression side of the beam $(\mathrm{D} / 50, \mathrm{RD} / 50$, $\mathrm{D} / 100, \mathrm{RD} / 100)$, the following findings have been obtained.

(a) As the influence of artificial cracks, on the compression face, the principal strain distribution due to compression and compressive collaspe is 
recognized. Also, the minimum principal strain corresponds to the artificially damaged region. On the tension face, the maximum principal strain distribution due to opening cracks is observed.

(b) As the effect of a repair, noticeable effect is not recognized on the tension face. The local distribution of the minimum principal strain changed from vertical to horizontal spreading, and that the effects of the repair are partly recognized about the minimum principal strain distribution in the artificially damaged region.

When the depth of artificially damaged region is full of the height (D/200 and $\mathrm{RD} / 200)$, the following findings have been obtained.

(a) As the influence of artificial cracks, on the tension face, a strong distribution due to a few cracks is seen. The distribution spreads diagonally at shallow angles, not spreading vertically. Also no clear change is observed across the boundary of the artificially damaged region. On the compression face, the minimum principal strain distribute around the loading plate takes place.

(b) As the effect of a repair, noticeable improvements are not observed in the maximum and minimum strain distribution, and no clear change is observed across the boundary of the artificially damaged region.

Since this paper is limited to analysis of a part of the captured image, detailed analysis of each load stage will be carried out in the future.

\section{Reference}

1. P. Deng, T. Matsumoto: Determination of dominant degradation mechanisms of RC bridge deck slabs under cyclic moving loads, International Journal of Fatigue, No 112, pp328-340 (2018)

2. S. Matsui: Road bridge deck: design, construction and maintenance, pp11-12, Morikita Publishing (2007)

3. T. Matsumoto, X. He: Image analysis of damage and fracture of CFRP box beams, The Fourteenth East Asia - Pacific Conference on Structural Engineering and Construction (EASEC-14), pp. 383-384 (2016) 